\title{
Reconciling Smiles for Index and Stock Options
}

\author{
Nicole Branger* \\ Holger Kraft $\ddagger$ \\ Antje Mahayni ${ }^{\S}$ \\ Christian Schlag
}

This version: March 18, 2008

\begin{abstract}
The fact that the implied volatility smiles for equity indices are strongly downward sloping, while the typical individual stock exhibits either a flat or even an upward sloping smile is sometimes considered puzzling. We show that this effect can easily be generated in a simple and parsimonious two-factor stochastic volatility model in the spirit of Bates (2000). From a theoretical perspective our paper adds to the literature by proving a 'diversification' result with respect to idiosyncratic stochastic volatility components of individual stocks, so that in the limit for infinitely many stocks, the prices of index options are solely based on the common volatility component. This result holds without restrictive assumptions on the structure of the correlations between stock returns and volatility changes. We employ Monte Carlo simulation to show that our model can reproduce the empirical observations quite well for an index of 30 stocks similar to the Dow Jones Industrial Average.
\end{abstract}

Keywords: Stochastic volatility, index options, equity options, volatility components

JEL: G11, G12

* Finance Center Münster, Westfälische Wilhelms-Universität Münster, 48143 Münster, Germany. E-mail: Nicole.Branger@wiwi.uni-muenster.de

$\ddagger$ Finance Department, Goethe University, D-60054 Frankfurt am Main, Germany. E-mail: kraft@finance.uni-frankfurt.de

$\S$ Mercator School of Management, University of Duisburg-Essen, D-47057 Duisburg, Germany. E-mail: antje.mahayni@uni-due.de

ฯ Finance Department, Goethe University, D-60054 Frankfurt am Main, Germany. E-mail: schlag@finance.uni-frankfurt.de 


\section{Introduction and Motivation}

The fact that the shapes of the implied volatility curves for the major equity market indices and the typical individual stocks are markedly different is sometimes seen as an important puzzle in the area of empirical option pricing research. For example, in a recent paper Bakshi, Kapadia, and Madan (2003) ask the following question: 'What causes the slope of the individual smiles to reverse its sign? The differential pricing in the cross section of strikes and in the cross section is puzzling.' (p. 123). The fact that implied volatilities for options on single stocks can be increasing in the strike price is of course in sharp contrast with the stylized empirical facts about index options, for which smiles are basically always downward sloping and, in addition, rather steep.

Still, there are several papers tackling this issue explicitly. For example, Buraschi, Trojani, and Vedolin (2008), also motivated by the above quote from Bakshi, Kapadia, and Madan (2003), develop a complex general equilibrium model with heterogeneous beliefs, which can actually produce different smile shapes for indices and individual stocks, and especially upward sloping smiles for stocks. On the other hand, it is not clear if to relate the upward sloping smiles for individual stocks to investors' optimism is indeed a fully satisfactory solution to the problem. The mechanism which ultimately produces this result is very complicated, and it is not easy to detect the factors in the model which are ultimately responsible for this outcome.

In this paper we propose a rather different line of argument. A relatively simple two-factor model in the spirit of Bates (2000) with a common and an idiosyncratic volatility factor is very well able to reproduce the structures that we see in the data. All that is necessary in our setup is a negative correlation between the common volatility factor and stock returns and the opposite relation for the idiosyncratic volatility component. We value index options as basket options, thereby taking into account the restriction that an index level is nothing but a weighted sum of individual 
stock prices. An important theoretical result we derive is that the idiosyncratic component of stochastic stock return volatility follows a 'diversification law' very similar to the well-known one for returns. As intuitive this result may seem, it turns out to be highly non-trivial and requires a full proof based on results from the convergence theory for stochastic processes. Since there is no closed-form solution for the price of an index option, given two-factor stochastic volatility models for individual stocks, we apply Monte Carlo simulation to value index options. This also represents a 'finite-sample' application of our theoretical result, and we show that even this simple model does a very good job in explaining the stylized facts in the data.

There are a couple of other papers, dealing either with differences between individual stocks and indices or time-varying slopes of implied volatility smiles. In a jump-diffusion setup Branger and Schlag (2004) decompose both types of risk factors into an idiosyncratic and a common part and show that it is the intensity for common jumps which controls the shape of the smile for index options relative to that for the typical individual stock. As a generalization of the model developed by Heston (1993), Bates (2000) suggests the above mentioned two-factor stochastic volatility model, which allows for a stochastic correlation between stock returns and volatility changes and thus makes the slope of the implied volatility smile stochastic as well. Carr and Wu (2007) and Bakshi, Carr, and Wu (2008) also take up the issue of a time-varying slope of the implied volatility smile and suggest, among other things, models based on Lévy processes.

The remainder of the paper is organized as follows. In Section 2 we introduce the model setup. In Section 3 we present the results of our Monte Carlo analysis for the valuation of index options. Section 4 concludes. 


\section{Model and Theoretical Results}

\section{$2.1 \quad$ Basic setup}

In our economy there are $n$ stocks which exhibit stochastic volatilities driven by two components as in the model suggested by Bates (2000). In detail, the price $S_{i}$ of stock $i$ and its two volatility components $V_{m}$ and $V_{i}$ satisfy the following system of stochastic differential equations under the risk-neutral measure $\mathbb{Q}$ :

$$
\begin{aligned}
d S_{t, i}= & S_{t, i}\left[r d t+\sqrt{V_{t, m}}\left(\rho_{i, m} d W_{t}^{m, 1}+\sqrt{1-\rho_{i, m}^{2}} d W_{t}^{m, 2}\right)\right. \\
& \left.+\sqrt{V_{t, i}}\left(\rho_{i, i} d W_{t}^{i, 1}+\sqrt{1-\rho_{i, i}^{2}} d W_{t}^{i, 2}\right)\right] \\
d V_{t, m}= & \kappa_{m}\left(\theta_{m}-V_{t, m}\right) d t+\sigma_{m} \sqrt{V_{t, m}} d W_{t}^{m, 1} \\
d V_{t, i}= & \kappa_{i}\left(\theta_{i}-V_{t, i}\right) d t+\sigma_{i} \sqrt{V_{t, i}} d W_{t}^{i, 1} .
\end{aligned}
$$

We will refer to $V_{m}$ as the common or market component of volatility, while $V_{i}$ will be called the idiosyncratic component. The two volatility components follow mean-

reverting square-root processes to guarantee non-negativity. $W^{m, 1}, W^{m, 2}, W^{i, 1}$, and $W^{i, 2}$ are independent standard Wiener processes.

It will turn out that the key parameters to control the slopes of the implied volatility curves for the index and the individual stocks in this model are the correlation coefficients $\rho_{i, m}$ and $\rho_{i, i} . \rho_{i, m}$ represents the correlation between the return on stock $i$ and the market volatility component, and $\rho_{i, i}$ is the analogous quantity for the idiosyncratic component. It is the signs of these two correlation coefficients which are ultimately responsible for the shape of the smiles for the index and the individual stocks. In detail a negative value for $\rho_{i, m}$ and a positive one for $\rho_{i, i}$ will lead to a relatively pronounced negative slope of the index smile, while the implied volatility curves for individual stocks will be flat or even upward sloping, depending on the numerical values for the two correlation coefficients and on the current realizations of the two volatility components. When the market volatility is high as 
compared to the idiosyncratic volatility, the correlation between the overall volatility and the return on the stock goes to $\rho_{i, m}$, and the smile is downward sloping. If the idiosyncratic volatility dominates, it is upward sloping.

In our approach the index level $I$ is represented as an equally weighted sum of the $n$ stock prices, i.e. $I_{t}=\frac{1}{n} \sum_{i=1}^{n} S_{t, i}$. While this is a somewhat simplifying assumption, the exact structure of the weights of individual stocks in the index formula is of minor relevance for the issue we are interested in. Intuitively, the overall volatility of the index is mainly driven by the global volatility component, while the impact of the idiosyncratic volatility components decreases when the number of stocks in the index increases. With the correlation between the global volatility and the returns on individual stocks being negative, the volatility smile for the index is downward sloping.

For the special case when all stocks and all idiosyncratic volatilities follow exactly the same process, in particular when the correlation between stock returns and the market and idiosyncratic volatility components are the same for all stocks, we explicitly derive two interesting limiting results when the number of stocks in the index becomes large. First, the idiosyncratic volatility components of the individual stocks vanish from the dynamics of the index, which is then solely driven by the common component. Second, also in the limit for $n$ tending to infinity, index option prices can be computed based on a Heston type model with only the common volatility component. Note that the above restrictions on the parameters still allow the parameters of the common component to be different from that of the idiosyncratic part of volatility, i.e. it is perfectly possible to consider a model with $\kappa_{m} \neq \kappa_{i}$ etc.

For the more general case with (almost) arbitrary parameters of the individual processes our results can be derived in a very similar fashion. This extension is basically straightforward, although the associated proofs would be rather lengthy, 
since one has to take into account the fact that the second moments of all processes would then be different. To provide as much intuition as possible, we therefore prefer to present the simpler proofs for the case of identical parameters.

\subsection{Diversification in the limit}

The first result concerns the limiting properties of the idiosyncratic volatility component (all proofs can be found in the appendix). Note that, compared to the initial model presentation, the Cholesky decomposition of the covariance matrix is represented the other way around here. This is, of course, immaterial for the results.

\section{Lemma 1 (Vanishing idiosyncratic volatility) Let}

$$
\begin{aligned}
& d S_{t, i}=S_{t, i}\left(r d t+\sqrt{V_{t, m}} d W_{t}^{m, 1}+\sqrt{V_{t, i}} d W_{t}^{i, 1}\right) \\
& d V_{t, j}=\kappa_{j}\left(\theta_{j}-V_{t, j}\right) d t+\sigma_{j} \sqrt{V_{t, j}}\left(\rho_{j} d W_{t}^{j, 1}+\sqrt{1-\rho_{j}^{2}} W_{t}^{j, 2}\right)
\end{aligned}
$$

for $j \in\{i, m\}(i=1, \ldots, n) . W^{j, k}(k=1,2, j \in\{i, m\})$ are Brownian motions with $\left\langle W^{i, k}, W^{j, l}\right\rangle=0$ for $i \neq j$ and/or $k \neq l$. Assume

$$
\rho_{j}<\frac{\kappa_{j}}{\sigma_{j} \omega}
$$

and

$$
\left(\rho_{j} \sigma_{j} \omega-\kappa_{j}\right)^{2}>\sigma_{j}^{3}\left(\omega^{2}-\omega\right)
$$

for $j=m, \omega=2$ and for $j=1, \omega=4$ respectively. Then

$$
\lim _{n \rightarrow \infty} E\left[\frac{1}{n^{2}} \int_{0}^{t} \sum_{i=1}^{n} S_{i u}^{2} V_{i u} d u\right]=0 .
$$

The lemma says that in the limit the idiosyncratic volatility components of the individual stocks no longer have an impact on the quadratic variation of the index level. A proof is given in the Appendix.

The implications of this result in terms of financial economics are far-reaching, since individual stock price processes can be (almost) arbitrarily parametrized to 
produce a wide variety of shapes for the implied volatility smile via the idiosyncratic component, while the index would always exhibit the smile that is generated by the common component. In that sense Lemma 1 opens up the possibility that the prices of index options are based on the common component only, while options on individual stocks are affected by both the common and the idiosyncratic part of volatility, so that they could basically exhibit different shapes of the smile, while the smile for index options would always remain the same based on the parameters for the common component.

\subsection{Index option pricing}

The following theorem establishes the key theoretical result of the paper by showing that the diversification result in Lemma 1 can be extended to option pricing.

Theorem 1 (Index put option pricing) Let the dynamics of the asset prices be given by the system (1), and let $I_{t}^{(n)} \equiv \frac{1}{n} \sum_{i=1}^{n} S_{t, i}$. Furthermore, assume that the conditions of Lemma 2 in the appendix are satisfied. Then it holds that

$$
\lim _{n \rightarrow \infty} E\left[\left(K-I_{T}^{(n)}\right)^{+}\right]=E\left[\left(K-I_{T}^{(\infty)}\right)^{+}\right]
$$

where

$$
\begin{aligned}
I_{t}^{(\infty)} & \equiv I_{0}^{(\infty)}+\int_{0}^{t} I_{u}^{(\infty)} r d u+\int_{0}^{t} I_{u}^{(\infty)} \sqrt{V_{u, m}} d W_{u}^{m, 1} \\
I_{0}^{(\infty)} & \equiv \lim _{n \rightarrow \infty} I_{0}^{(n)}
\end{aligned}
$$

Note that the theorem states the result for put options, but put-call parity of course assures the corresponding fact for calls. In reality one would furthermore never be able to observe an index based on an infinite number of stocks. Nevertheless, the result gives a hint what to expect in terms of indices with different numbers of stocks. The larger this number, the more pronounced index option prices (or, more 
precisely, the shape of the smile) will potentially differ from the smiles of individual stocks.

\section{Simulation Analysis}

In this section we will analyze the quantitative output of the model. While the prices of options on the individual stocks can be computed in closed form using the formula developed by Bates (2000), this is not possible for the index as a basket of stocks. This is not a special property of our model, since even in the simplest possible case of geometric Brownian motions for the individual stocks, the index would not be lognormally distributed (except in a situation where all stocks are perfectly positively correlated) and options on it would have to be priced numerically.

We therefore discretize the stochastic processes for the individual stocks using a simple Euler scheme, which has proved to be quite robust and reliable in simulations with small time increments (see, e.g., Christoffersen, Jacobs, and Mimouni (2007). We consider options with a maturity of three months. The number of trading days per year is assumed to be 252 , so that the chosen time to maturity represents 63 trading days. The dynamics of the prices and volatilities are simulated assuming 10 time steps per day, so that the discretization error is likely to be rather small. Should nevertheless a value less than $10^{-4}$ be drawn for one of the variance processes, its value would be mirrored at this boundary level of $10^{-4}$. All the results are based on 100,000 simulation runs. The index is composed of 30 stocks and thus resembles the Dow Jones Industrial rather closely. At the same time, our index avoids the major deficiencies of the Dow, since in our case all stocks have the same initial price $\left(S_{0, i}=100\right.$ for $\left.i=1, \ldots, 30\right)$, and there are no dividends.

In our base scenario the parameters are given by $\theta_{m}=0.08, \kappa_{m}=2, \sigma_{m}=0.4$,

$\rho_{m}=-0.8$ for the market component and by $\theta_{i}=0.08, \kappa_{i}=2, \sigma_{i}=0.4, \rho_{i}=0.8$ 
for the idiosyncratic component. The results for this setting are shown in Figure 1, where implied volatility is plotted as a function of moneyness $M$ (defined as strike price divided by stock price or index level, respectively). The option prices for the individual stocks (and thus, implicitly also the volatility smile) are calculated by Fourier inversion, while the smile for the index is computed via a Monte Carlo simulation of 30 stocks, all of which exhibit the dynamics represented by the parameter set just described.

Like in the Heston (1993) model, the slope of the smile in the two-factor model is driven by the correlation between volatility changes and returns on the underlying. In the two-factor case there are two such correlations, and their weights in the overall correlation depend on the current relative magnitude of the two volatilities. So when $V_{0, i}$ is large relative to $V_{0, m}$, then the positive correlation $\rho_{i, i}$ associated with $V_{i}$ will dominate and induce a slightly upward sloping smile. The value for the implied volatility of the equity option is 0.351 for a moneyness of $M=0.8$ and 0.369 for $M=1.2$. In between the implied volatilities first decrease until roughly $M=0.92$ and then increase monotonically. Since the empirically relevant range of strikes is certainly located to the right of 0.92 , one would observe a strictly upward sloping implied volatility curve for the typical individual stock. On the other hand, the index exhibits a pronounced negative slope in its smile. Implied volatility falls from almost $30 \%$ for $M=0.8$ to around $17 \%$ for $M=1.2$. so the model re-produces the patterns observed in empirical studies qualitatively quite well.

One may ask if the values chosen for the correlation coefficients $\rho_{i, i}$ and $\rho_{i, m}$ are not too extreme. Absolute values of 0.8 can certainly be considered large, but the main purpose of our paper is to match the qualitative properties of the respective smile curves with a rather parsimonious model, rather than to focus on a perfect calibration of smiles for different maturities, for which jumps would be an absolute necessity. So when stochastic volatility alone is to produce a certain steepness in the 
index smile, a larger value in absolute terms for the correlation $\rho_{i, m}$ is needed, and in turn $\rho_{i, i}$ must also be larger to compensate for this.

Figures 2, 3, and 4 show the smile curves for variations of the basic scenario, where the starting values for the idiosyncratic volatility component $V_{0, i}$ and the correlation coefficients $\rho_{i, i}$ and $\rho_{i, m}$ need not be the same for all 30 stocks anymore. To avoid a systematic influence of our choice of parameters, we randomly assigned parameter values to the individual stocks by sampling from a uniform distribution for the given parameter(s). The index is then computed on the basis of these 30 simulated stock prices. In the graphs, however, the curves for the individual stocks are those for the representative parameter values described in the caption of the respective figure, to make the pictures easier to interpret.

First, we vary the initial value of idiosyncratic volatility by sampling $V_{0, i}$ from a uniform distribution over the interval $\left[0.5 \theta_{i} ; 1.5 \theta_{i}\right]$. As one can see immediately from Figure 2, different values for $V_{0, i}$ mostly change the level of the individual implied volatility curves, but leave the general findings unchanged. Again, when the market component is small relative to the (now different) $V_{i}$, the individual smiles are upward sloping, while the index exhibits the usual picture. When $V_{0, m}$ becomes larger, the smile for individual stocks becomes symmetric and eventually downward sloping. The index nevertheless retains a smile, which is in downward sloping in a much more pronounced fashion than the one for the individual stocks.

Adding even more flexibility to the model by allowing the correlation coefficients $\rho_{i, i}$ and $\rho_{i, m}$ to differ across stocks yields the pictures shown in Figures 3 and 4. The variation in $\rho_{i, i}$ was generated by sampling its value form a uniform distribution over $[0 ; 0.8]$, while the correlation with the common component was drawn from the interval $[-0.8 ; 0]$. These choices naturally limit the variation in individual and index smiles that the model can produce. However, we consider it most important to conserve the downward sloping index smile, while at the same time allowing for a 
wide variety of smile shapes for the individual stocks. The graphs in the two figures confirm that our model offers a parsimonious explanation for the fact of different smile shapes for indices and individual stocks. Of course, the effects are now slightly less pronounced than in the original case where all correlations were chosen at the extreme values of -0.8 and 0.8 . Nevertheless, one obtains qualitatively the same results as in the previous scenarios.

The main message from the graphs is that the individual stocks comprising the index can be rather heterogeneous with respect to their dynamics, and even exhibit upwards sloping smiles. As long as the correlations between stock returns and the changes in the two volatility components have certain signs (and certain critical magnitudes), the index will still have a downward sloping smile curve.

\section{Conclusion}

Index options are usually priced in basically the same way as options on a stock, namely by assuming certain dynamics for the index level and potential state variables like stochastic volatility. The index is, however, not given exogenously, its value is derived from the prices of the stocks included. This fact becomes important when one studies structural differences between stock and index options, since the dynamics of the index are determined by the joint distribution of the stocks, and not the other way around.

In this paper we have dealt with the (seemingly) puzzling empirical observation that the implied volatility curves for indices are usually heavily sloped downwards, while the smile curve for the typical stock is either rather flat or even upward sloping. We have shown that this effect can result from a parsimonious two-factor stochastic volatility model in the spirit of Bates (2000), where the key parameters turn out to be the correlation coefficients of the two variance processes with the return on the stock. 
In case the correlation with the common volatility component is negative, while the one with the idiosyncratic component is positive, we will obtain qualitatively exactly the results documented in the empirical option pricing literature. Of course, since we do not consider jumps in our model, we are not able to fit the smile perfectly, but this extension seems rather straightforward.

In a next step it would be interesting to estimate the model empirically, based on a set of individual stocks as well as an associated index to see how much the correlation coefficients between stock returns and variance changes actually vary cross-sectionally and if a common variance component can be identified in the data. 


\section{A Proofs}

\section{A.1 Proof of Lemma 1}

The following lemma will turn out to be useful in the proofs of the main results of the paper.

\section{Lemma 2 (Andersen/Piterbarg (2007)) Let}

$$
\begin{aligned}
d X_{t} & =X_{t}\left(r d t+\sqrt{V_{t}} d W_{t}^{(1)}\right) \\
d V_{t} & =\kappa\left(\theta-V_{t}\right) d t+\sigma \sqrt{V_{t}}\left(\rho d W_{t}^{(1)}+\sqrt{1-\rho^{2}} d W_{t}^{(2)}\right)
\end{aligned}
$$

where the conditions

$$
\rho<\frac{\kappa}{\sigma \omega}
$$

and

$$
(\rho \sigma \omega-\kappa)^{2}>\sigma^{3}\left(\omega^{2}-\omega\right)
$$

are satisfied. Then

$$
E\left[\left(X_{T}\right)^{\omega}\right]<\infty
$$

Remark. In their Proposition 3.1, Andersen and Piterbarg (2007) show that sufficient conditions are given by $a<0$ and $D>0$ where

$$
\begin{aligned}
a & \equiv \frac{2(\rho \sigma \omega-\kappa)}{\sigma^{2}} \\
D & \equiv a^{2}-4 b
\end{aligned}
$$

with $b \equiv \frac{\omega(\omega-1)}{\sigma}$.

We are now going to prove Lemma 1. Observe that, by Fubini's theorem,

$$
\begin{aligned}
& \lim _{n \rightarrow \infty} E\left[\frac{1}{n^{2}} \int_{0}^{t} \sum_{i=1}^{n} S_{u, i}^{2} V_{u, i} d u\right] \\
= & \lim _{n \rightarrow \infty} \frac{1}{n^{2}} \int_{0}^{t} \sum_{i=1}^{n} E\left[S_{u, i}^{2} V_{u, i}\right] d u \\
= & \lim _{n \rightarrow \infty} \frac{1}{n^{2}} n \int_{0}^{t} E\left[S_{u, 1}^{2} V_{u, 1}\right] d u \\
= & \lim _{n \rightarrow \infty} \frac{1}{n} \int_{0}^{t} E\left[S_{u, 1}^{2} V_{u, 1}\right] d u .
\end{aligned}
$$


Therefore, it is sufficient to show that

$$
E\left[S_{u, 1}^{2} V_{u, 1}\right]<\infty
$$

Since

$$
d S_{t, i}=S_{t, i}\left(r d t+\sqrt{V_{t, m}} d W_{t}^{m, 1}+\sqrt{V_{t, i}} d W_{t}^{i, 1}\right)
$$

it follows that

$$
S_{t, i}=S_{0,1} \exp \left\{\int_{0}^{t}\left(r-\frac{1}{2}\left(V_{u, m}+V_{u, i}\right)\right) d u+\int_{0}^{t} \sqrt{V_{u, m}} d W_{u}^{m, 1}+\int_{0}^{t} \sqrt{V_{u, i}} d W_{u}^{i, 1}\right\} .
$$

Defining

$$
\begin{aligned}
X_{t}^{m} & \equiv \exp \left\{\int_{0}^{t}\left(r-\frac{1}{2} V_{u, m}\right) d u+\int_{0}^{t} \sqrt{V_{u, m}} d W_{u}^{m, 1}\right\} \\
X_{t}^{i} & \equiv \exp \left\{\int_{0}^{t}-\frac{1}{2} V_{u, i} d u+\int_{0}^{t} \sqrt{V_{u, i}} d W_{u}^{i, 1}\right\}
\end{aligned}
$$

gives (for $i=1$ )

$$
S_{t, 1}^{2} V_{t, 1}=S_{0,1}^{2}\left(X_{t}^{m}\right)^{2}\left(\left(X_{t}^{1}\right)^{2} V_{t, 1}\right)
$$

where $\left(X_{t}^{m}\right)^{2}$ and $\left(X_{t}^{1}\right)^{2} V_{1 t}$ are independent. This implies

$$
\begin{aligned}
E\left[S_{t, 1}^{2} V_{t, 1}\right] & =S_{10}^{2} E\left[\left(X_{t}^{m}\right)^{2}\right] E\left[\left(X_{t}^{1}\right)^{2} V_{1 t}\right] \\
& \leq S_{0,1}^{2} E\left[\left(X_{t}^{m}\right)^{2}\right]\left(E\left[\left(X_{t}^{1}\right)^{4}\right] E\left[\left(V_{t, 1}\right)^{2}\right]\right)
\end{aligned}
$$

Thus, the existence of the second moment of $X_{t}^{m}$, the fourth moment of $X_{t}^{1}$ and the second one of $V_{t, 1}$ are sufficient conditions for $E\left[S_{u, 1}^{2} V_{u, 1}\right]<\infty$. Notice that $V_{t, 1}$ is a Bessel process such that the existence of the second moment is given. The rest of the proof follows by Lemma 2 and the conditions stated there. 


\section{A.2 Proof of Theorem 1}

Consider first the following chain of equalities:

$$
\begin{aligned}
d I_{t}^{(n)} & =d\left(\frac{1}{n} \sum_{i=1}^{n} S_{t, i}\right) \\
& =\frac{1}{n} \sum_{i=1}^{n} d S_{t, i} \\
& =\frac{1}{n} \sum_{i=1}^{n} S_{t, i}\left(r d t+\sqrt{V_{t, m}} d W_{t}^{m, 1}+\sqrt{V_{t, i}} d W_{t}^{i, 1}\right) \\
& =I_{t}^{(n)}\left(r d t+\sqrt{V_{t, m}} d W_{t}^{m, 1}\right)+\frac{1}{n} \sum_{i=1}^{n} S_{t, i} \sqrt{V_{t, i}} d W_{t}^{i, 1} .
\end{aligned}
$$

Using $\left\langle W^{i, k}, W^{j, l}\right\rangle=0$ for $i \neq j$ and/or $k \neq l$, we have

$$
d\left\langle I^{(n)}\right\rangle_{t}=\left(I_{t}^{(n)}\right)^{2} V_{t, m} d t+\frac{1}{n^{2}} \sum_{i=1}^{n} S_{t, i}^{2} V_{t, i} d t
$$

By a result in Protter (2005) (p. 271), the sequence $\left\{I^{(n)}\right\}_{n}$ thus converges to $I^{(\infty)}$ uniformly on compacts in probability (ucp) if the process $Y^{(n)}$ defined by $Y_{t}^{(n)} \equiv \frac{1}{n} \sum_{i=1}^{n} \int_{0}^{t} S_{t, i} \sqrt{V_{t, i}} d W^{i, 1}$ converges to zero in the semimartingale topology. This is valid if $Y^{(n)}$ converges to zero prelocally in $\underline{\underline{H}}^{2}$. This follows from Lemma 1 where we have shown that

$$
\lim _{n \rightarrow \infty} E\left[\left(\frac{1}{n} \int_{0}^{t} \sum_{i=1}^{n} S_{u, i} \sqrt{V_{u, i}} d W_{u}\right)^{2}\right]=\lim _{n \rightarrow \infty} E\left[\frac{1}{n^{2}} \int_{0}^{t} \sum_{i=1}^{n} S_{u, i}^{2} V_{u, i} d u\right]=0 .
$$

The rest of the claim then follows from the the portmanteau theorem of weakconvergence theory (see, e.g., Billingsley (1968)). 


\section{References}

Andersen, L., and V. Piterbarg, 2007, Moment explosions in stochastic volatility models, Finance and Stochastics 11, 29-50.

Bakshi, G., P. Carr, and L. Wu, 2008, Stochastic risk premiums, stochastic skewness in currency options, and stochastic discount factors in international economies, Journal of Financial Economics 87, 132-156.

Bakshi, G., N. Kapadia, and D. Madan, 2003, Stock Return Characteristics, Skew Laws, and the Differential Pricing of Individual Equity Options, Review of Financial Studies 16, 101-143.

Bates, D., 2000, Post-'87 Crash Fears in the S\&P 500 Futures Option Market, Journal of Econometrics 94, 181-238.

Billingsley, P., 1968, Convergence of probability measures. (Wiley New York).

Branger, N., and C. Schlag, 2004, Why is the Index Smile So Steep?, Review of Finance 8, 109-127.

Buraschi, A., F. Trojani, and A. Vedolin, 2008, The Joint Behavior of Credit Spreads, Stock Options and Equity Returns When Investors Disagree, Working Paper.

Carr, P., and L. Wu, 2007, Stochastic Skew for Currency Options, Journal of Financial Economics 86, 213-247.

Christoffersen, P.F., K. Jacobs, and K. Mimouni, 2007, Models for S\&P 500 Dynamics: Evidence from Realized Volatility, Daily Returns, and Option Prices, Working Paper.

Heston, S.L., 1993, A Closed-Form Solution for Options With Stochastic Volatility with Applications to Bond and Currency Options, Review of Financial Studies 6, $327-343$. 
Protter, P.E., 2005, Stochastic Integration and Differential Equations. (Springer Berlin) 2nd edn. 

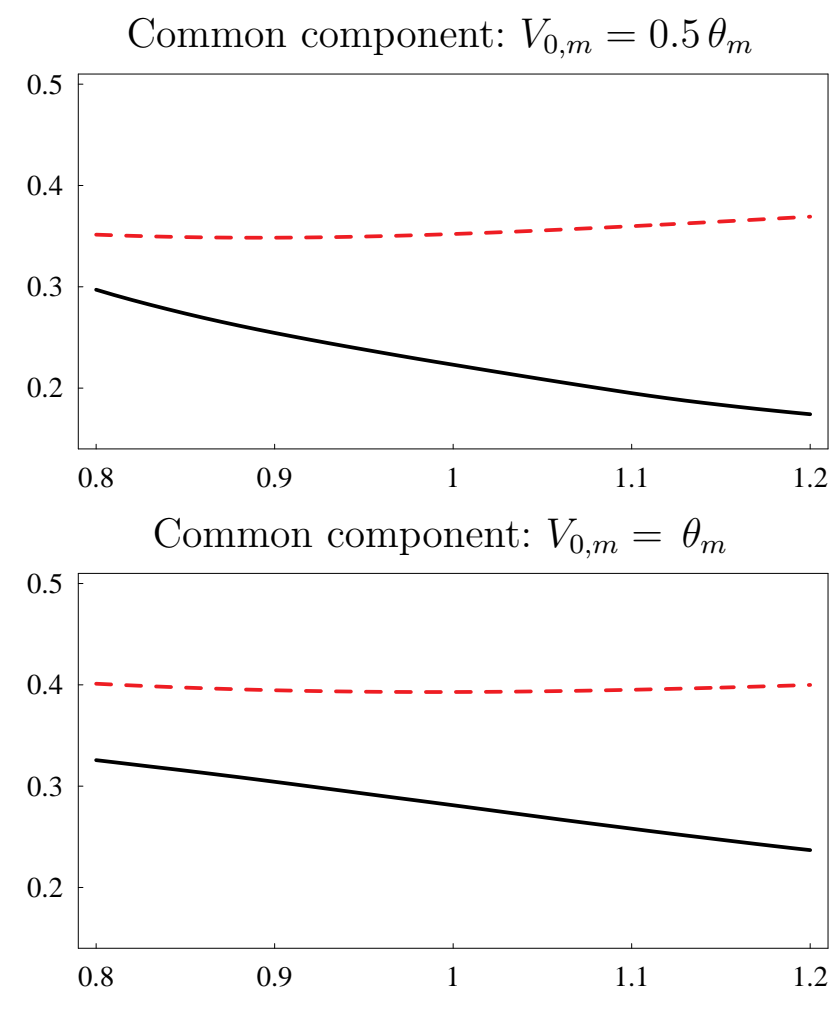

Common component: $V_{0, m}=1.5 \theta_{m}$

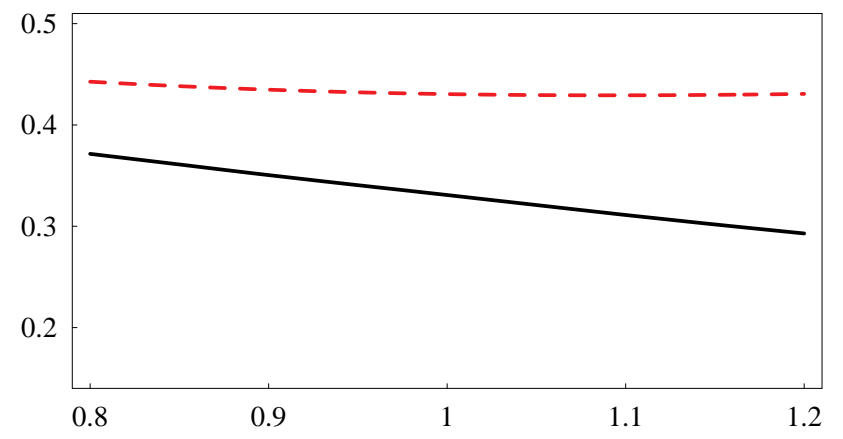

Figure 1:

\section{Base Case}

The graphs show the implied volatility smiles for the index (solid line) and for individual stocks (dashed line) as a function of moneyness (defined as strike price divided by stock price or index level, respectively). All individual stocks have the same dynamics.

The parameters are $\theta_{m}=0.08, \kappa_{m}=2, \sigma_{m}=0.4, \rho_{i, m}=-0.8$ for the common component, and $\theta_{i}=0.08, \kappa_{i}=2, \sigma_{i}=0.4, \rho_{i, i}=0.8$ for the idiosyncratic component. The initial values of the idiosyncratic variance processes are set equal to their mean-reversion level, i.e. $V_{0, i}=\theta_{i}$, and we consider three cases for the starting value $V_{0, m}$ of the common component: $V_{0, m}=0.5 \theta_{m}$ (upper graph), $V_{0, m}=\theta_{m}$ (middle graph) and $V_{0, m}=1.5 \theta_{m}$ (lower graph). The interest rate is set to zero. 

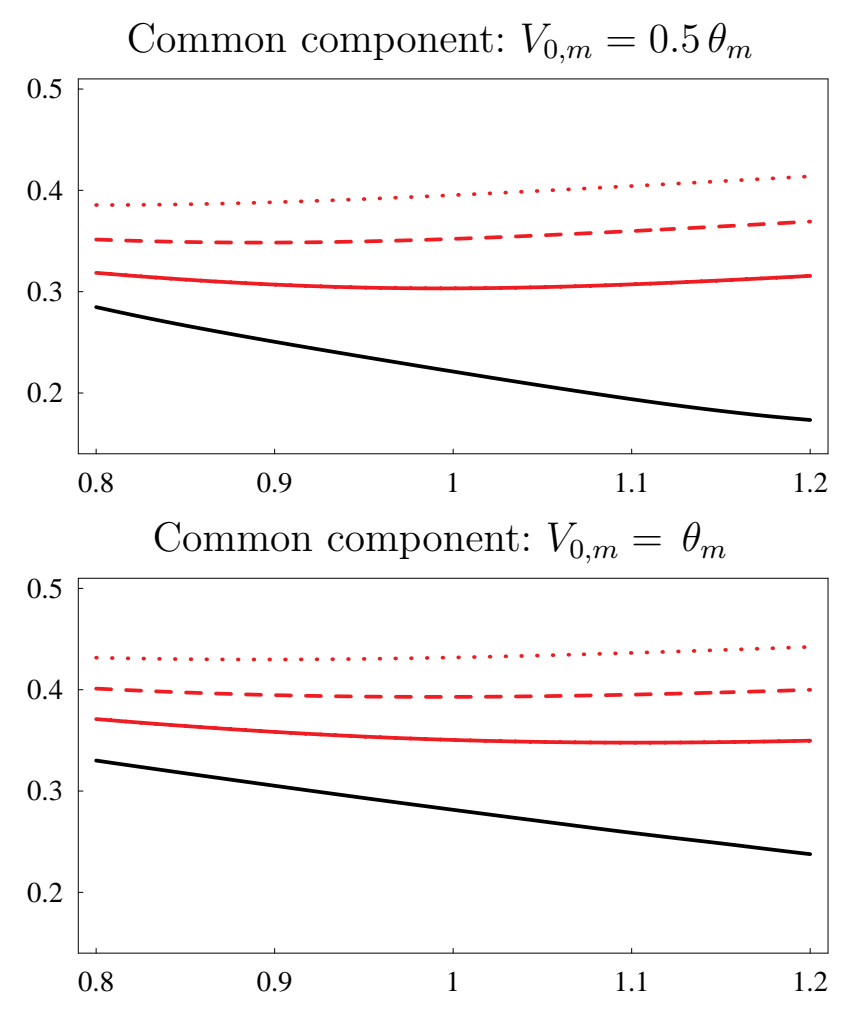

Common component: $V_{0, m}=1.5 \theta_{m}$

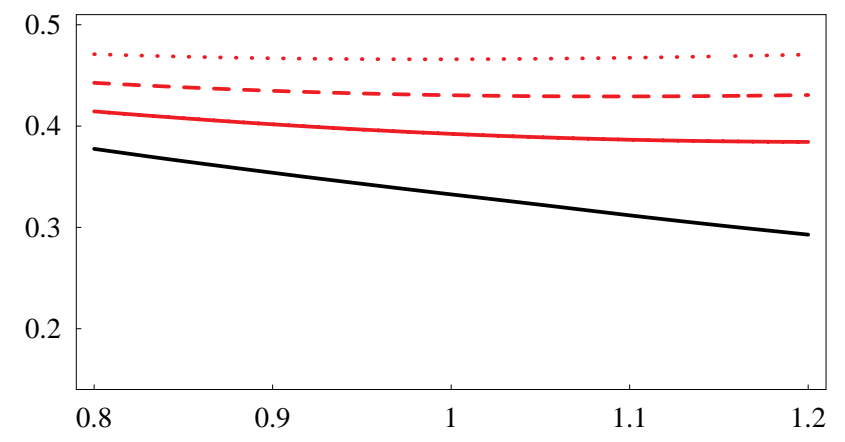

Figure 2:

\section{Different Initial Idiosyncratic Volatilities}

The graphs show the implied volatility smiles as a function of moneyness for the index (lower solid line) and for a representative set of individual stocks with different starting values for the idiosyncratic variance component (upper lines).

The parameters are $\theta_{m}=0.08, \kappa_{m}=2, \sigma_{m}=0.4, \rho_{i, m}=-0.8$ for the market component, and $\theta_{i}=0.08, \kappa_{i}=2, \sigma_{i}=0.4, \rho_{i, i}=0.8$ for the idiosyncratic component. The initial values $V_{0, i}$ of the idiosyncratic variance component are $0.5 \theta_{i}$ (upper solid line), $\theta_{i}$ (dashed line) and $1.5 \theta_{i}$ (dotted line). We consider three cases for the starting value $V_{0, m}$ of the common component: $V_{0, m}=0.5 \theta_{M}$ (upper graph), $V_{0, m}=\theta_{M}$ (middle graph) and $V_{0, m}=1.5 \theta_{M}$ (lower graph). The interest rate is set to zero. 

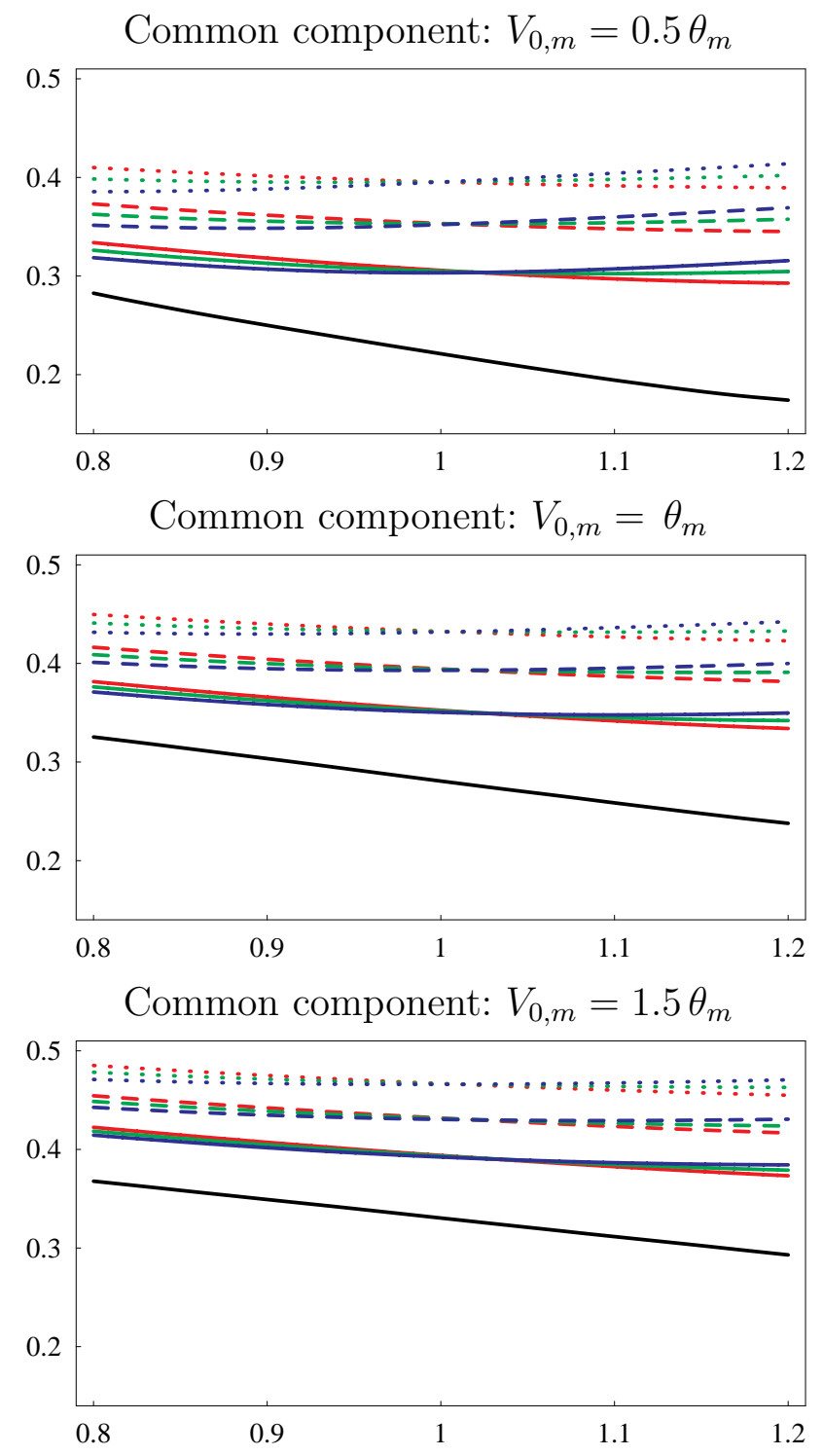

Figure 3:

\section{Different Initial Idiosyncratic Volatilities and Different Correlations with Idiosyncratic Volatility}

The graphs show the implied volatility smiles as a function of moneyness for the index (lower solid line) and for a representative set of individual stocks with different starting values for the idiosyncratic variance component and different correlation coefficients $\rho_{i, i}$ (upper lines).

The parameters are $\theta_{m}=0.08, \kappa_{m}=2, \sigma_{m}=0.4, \rho_{i, m}=-0.8$ for the market component, and $\theta_{i}=0.08, \kappa_{i}=2$, and $\sigma_{i}=0.4$ for the idiosyncratic component. The initial values $V_{0, i}$ of the idiosyncratic variance component are equal to $0.5 \theta_{i}, \theta_{i}$ and $1.5 \theta_{i}$. The correlation $\rho_{i, i}$ is equal to $0,0.4$, and 0.8 , respectively. We consider three cases for the starting value $V_{0, m}$ of the common component: $V_{0, m}=0.5 \theta_{m}$ (upper graph), $V_{0, m}=\theta_{m}$ (middle graph) and $V_{0, m}=1.5 \theta_{m}$ (lower graph). The interest rate is set to zero. 


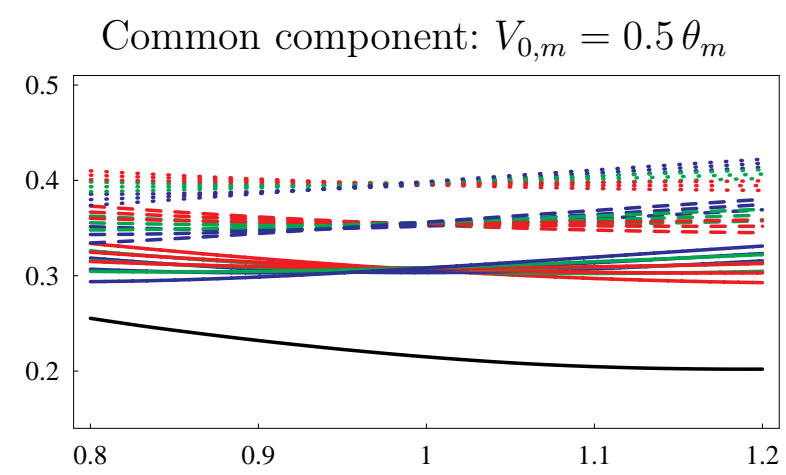

Common component: $V_{0, m}=$ thet $_{m}$

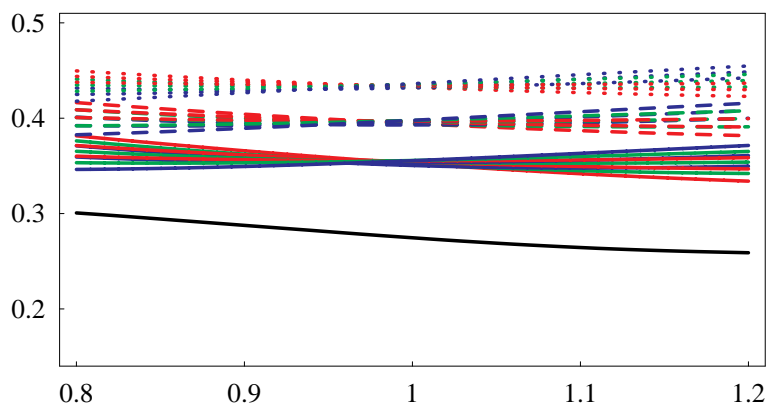

Common component: $V_{0, m}=1.5 \theta_{m}$

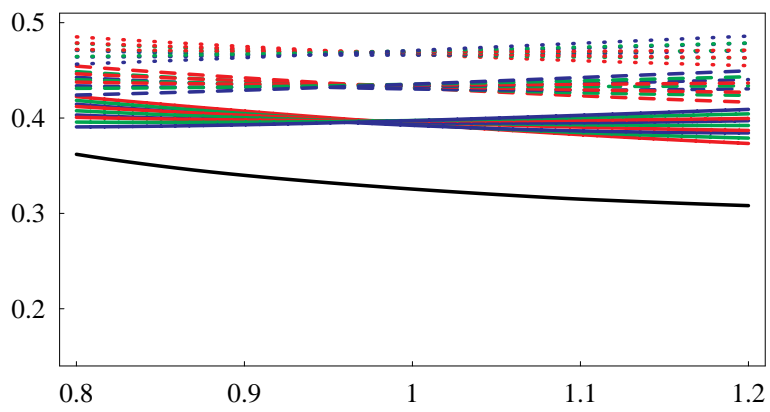

Figure 4:

\section{Different Initial Idiosyncratic Volatilities, Different Correlations with Idiosyncratic Volatility, Different Correlations with Market Volatility}

The graphs show the implied volatility smiles as a function of moneyness for the index (lower solid line) and for a representative set of individual stocks with different starting values for the idiosyncratic variance component and different correlation coefficients $\rho_{i, i}$ and $\rho_{i, m}$ (upper lines). The parameters are $\theta_{m}=0.08, \kappa_{m}=2, \sigma_{m}=0.4, \rho_{i, m}=-0.8$ for the market component, and $\theta_{i}=0.08, \kappa_{i}=2$, and $\sigma_{i}=0.4$ for the idiosyncratic component. The initial values $V_{0, i}$ of the idiosyncratic variance component are equal to $0.5 \theta_{i}, \theta_{i}$ and $1.5 \theta_{i}$. The correlation $\rho_{i, i}$ is equal to $0,0.4$, and 0.8 , respectively, while $\rho_{i, m}$ is either $0,-0.4$, or -0.8 . We consider three cases for the starting value $V_{0, m}$ of the common component: $V_{0, m}=0.5 \theta_{m}$ (upper graph), $V_{0, m}=\theta_{m}$ (middle graph) and $V_{0, m}=1.5 \theta_{m}$ (lower graph). The interest rate is set to zero. 